\title{
Surgical Informed Consent Revisited: Time to Revise the Routine?
}

\author{
Kinga B. Skowron ${ }^{1,2} \cdot$ Peter Angelos ${ }^{1,2}$
}

Published online: 16 September 2016

(C) Société Internationale de Chirurgie 2016

\section{Surgical informed consent}

All surgery carries risk. Both surgeon and patient enter into an agreement acknowledging a certain level of risk for a belief in benefit. The surgeon, with considerably more experience on the subject, proposes the operation. The patient, often stressed by a life-changing diagnosis, consents to proceed. Upon hearing about risks of surgery, many patients would rather "die trying" than do nothing [1]. Patients commonly trust that the surgeon will achieve the best possible outcome and agree to proceed with surgery.

Every operation has a known possibility of complication, which cannot be completely prevented even in the best of hands. A complication can be defined as "any undesirable, unintended, and direct result of an operation affecting the patient, which would not have occurred had the operation gone as well as could reasonably be hoped" [2]. Despite a careful process of informed consent, surgeons can never convey the full scope of possible complications to a patient who is considering surgery.

Every surgeon can recall a case when a patient agreed to a high-risk emergency operation, suffered a complication, and after only a few days in the intensive care unit, the patient or the family requested to withdraw treatment. The surgeon is then faced with a difficult situation. Should he or

Kinga B. Skowron

kinga.skowron@uchospitals.edu

1 Department of Surgery, University of Chicago Medicine, 5841 S. Maryland Ave., MC 6040, Chicago, IL 60637, USA

2 MacLean Center for Clinical Medical Ethics, University of Chicago Medicine, Chicago, IL, USA she acquiesce to this request to withdraw treatment? The scenario raises an important question: Was consent truly "informed" in such a case? Has the surgeon somehow failed, not in the technical aspects of the operation, but in the communication with the patient? In the following paragraphs, we will explore the forces which may lead to such complicated situations.

\section{Patient perspective}

The relationship between surgeons and patients has changed dramatically, with a shift from a culture of paternalism in which the "doctor knows best" to one of respect for patient autonomy [3]. Shared decision-making models of the doctor-patient relationship are frequently considered the gold standard today. Patients are provided with the necessary information about the proposed operation and, with the expertise of their physician who incorporates their wishes into a recommendation, come to a decision for a plan of care which is ideally personalized to the patient's individual needs.

However, informed consent in its current form does not meet the needs of the shared decision-making model. The routine discussion of this process produces an illusion in which both the doctor and patient believe that all possible factors ("all risks, benefits, and alternatives") have been discussed and agreed upon [3]. Yet, many patient needs are not met by the current format of informed consent.

In practice, surgeons do not provide patients with all possible information and leave out perhaps critically relevant information. In a study of informed consent for highrisk surgery, only $45 \%$ of discussions met all elements of 
adequate informed consent, and $23 \%$ did not meet even basic elements [4]. In fact, the majority of patients have a poor understanding of what exactly is involved in postoperative care [5]. Surgeons rarely discuss specific postoperative interventions which are often needed and rarely elicit the patient's opinion regarding pursuing these treatments.

In a survey of patients undergoing an angiogram, Deber et al. [6] outlined the types of decisions which patients feel require their input. For decisions involving a single correct answer or those requiring expert knowledge, such as whether a medical test should be performed, patients defer the decision to their physician. However, with regard to decisions placing value on risks and benefits, patients prefer a shared decision-making model, in which a physician provides information and a decision is reached in partnership with the doctor. Therefore, if the need for invasive treatments that can drastically alter quality of life post-operatively is anticipated, these potential treatments should ideally be discussed during the informed consent conversation.

Furthermore, the informed consent process is necessary, but not sufficient to help a patient come to a decision regarding surgery. In a study of patients undergoing an elective operation, authors discovered that patients enter the conversation with a broad perspective of the surgeon and surgery in general, ranging from distrustful to unquestioning [7]. Before consenting, the patients describe a "leap to trust," in which their personal fears are consciously set aside. The informed consent process today does not address the unspoken patient concerns and may also not adequately acknowledge the importance of trust in the surgeon that surgical informed consent requires. By explicitly asking patients about their expectations for the procedure (for example, "to be rid of pain," or "to come home to my family"), we may better understand whether the patient's expectations fall within the scope of possible post-operative outcomes. If we explicitly uncover such expectations, we may avoid a situation in which the patient expects the surgeon to achieve a perhaps unattainable goal, which may lead to patient dissatisfaction.

Once they have agreed to proceed, patients also consent to certain elements of post-operative care. In the setting of critical illness, patients are willing to accept invasive and burdensome treatments if the likelihood of recovery is high [8]. The factors considered by patients to be important are variable and personal, but include pain and symptom management, and being treated like a "whole person" [9]. These values may be in conflict with surgeons' goals of preventing or defeating death, a "covenant of care" which they believe the patient to implicitly enter preoperatively [10]. However, there is great variability among patients in how much they prefer to limit the extent of certain treatments, which they commonly do not discuss with their surgeon preoperatively [11]. This discrepancy highlights a serious defect in our process of informed consent.

\section{Surgeon perspective}

Surgeons may find it difficult to perform all elements of the idealized informed consent process. One reason may be the time required for the process. In urgent situations, surgeons may unconsciously put undo pressure on the patient and their family by using persuasive language ("this should be done"), causing a patient who may prefer to forgo surgery to acquiesce under pressure. On the other hand, physicians may "hang the crepe" when they believe a positive outcome to be unlikely $[12,13]$. In this scenario, the surgeon may provide an overly pessimistic view of the post-operative course. If this prediction comes true, they will be correct; if it does not come true and the patient does well, the surgeon will be perceived as a hero. These common traps are examples of poor communication vices which may unduly influence a patient's decision in the consent process.

Due to the very active role which surgeons play in their patients' care, it is difficult not to see a complication as a personal error. As the sociologist Bosk famously summarized, when a patient of a surgeon dies, his colleagues ask not "What happened?" but rather "What did you do?" [14] Surgeons are inherently biased toward the potential benefit of a given intervention, which influences their approach to patient wishes and may lead to disagreement [15]. Simply assuming that the patient is in agreement with the "covenant of care" may result in conflict [16]. By accepting the covenant of care, surgeons accept their patients' trust and take it upon themselves to defend the patient, employing the ethical principles of beneficence and non-maleficence.

However, a patient's decision post-operatively to stop treatment conflicts with the covenant, leading surgeons to often employ paternalism and violate the patient's autonomous choices [16]. Physicians are justified to do so when the request is unreasonable, for example, when post-operative intubation was precautionary and the likelihood of extubation and successful recovery is essentially guaranteed. However, when the likelihood of recovery is uncertain or low, surgeons cannot be justified to protect the patient's life above all else, when the value of "meaningful recovery" must be defined by the patient or the patient's surrogate.

Surgeon-patient expectations are typically well aligned in the case of routine, low-risk surgery. However, when a patient is faced with a high likelihood of death if they do not proceed with a high-risk operation, expectations for the post-operative course may differ dramatically. A surgeon may see surviving surgery as a success, while a patient expects to return to their pre-operative functional status and 
live at home. How surgeons attempt to align these expectations was studied in a thought-provoking work by Schwarze et al. [17], in which surgeons performing high-risk procedures were interviewed regarding advance directives of their patients prior to surgery. The authors describe the concept of "surgical buy-in," in which surgeons disclose the potential need for extraordinary measures post-operatively and negotiate an agreement with the patient to participate in these treatments. In some instances, physicians revert to paternalism and deny patients' requests to limit post-operative care, which they believe to be included in a "contract" for the entire course of care. How or whether the surgeon achieves the patient's agreement with this "contract" is unclear.

Surgical error further complicates post-operative decision-making. Complications may occur in the absence of error, if all appropriate precautions were taken to minimize the risk of complication but a complication nevertheless occurred [18]. However, some estimates suggest that nearly one half of all surgical patients will suffer an error during the course of their post-operative care, which will frequently lead to additional errors and an increased length of hospitalization [19]. In a large survey of US surgeons performing high-risk surgery, Schwarze et al. [20] found that $63 \%$ of surgeons would not honor a patient's request to withdraw care post-operatively. Among factors which influence this decision were surgeon error and elective versus emergency operation. Thus, surgeon error in the setting of a complication raises the likelihood of conflict with patient decisions to withdraw care and increases a surgeon's likelihood to default to paternalism.

When an error results in a complication, the surgeon may feel significant guilt about the patient's situation. To place such a tremendous level of responsibility upon the surgeon, rather than to accept physician error as a part of the expected complication risk in modern medicine, is an important obstacle for surgical culture [21, 22]. Major medical errors are associated with a decrease in surgeon quality of life and increased rates of burnout [23]. The common surgical culture of accepting full responsibility for patients and their outcomes, while honorable, may be paradoxically harmful. While errors will inevitably happen despite improvements in system-based safety nets, improving communication with patients and bridging the gap in expectations between doctor and patient will help not only patients, but may improve surgeon satisfaction as well.

\section{Suggestions for improvement}

Despite the seeming lack of time, simple communication tools can be used to encourage conversation with the patient. Physical cues, such as eye contact, hand contact, and sitting during conversation can help to create an atmosphere of trust and openness. Simply asking "How do you feel about this?" can help patients to express their concerns and to build trust in their surgeon [7]. Empathy and validation of the patient's fears can be perhaps the most important communication tools during this process.

While patients trust their surgeon to perform the operation, they may be entering the agreement with unreasonable expectations of the surgeon for the post-operative outcome. If these expectations are not met, the surgeon may feel guilt at having failed the patient. Therefore, an explicit discussion of the patient's expectations and whether these can reasonably be achieved by surgery is critical. A novel process for incorporating this discussion in the informed consent was recently proposed by Kruser et al. [24]. In this model, patients are provided with a visual drawing of the options available, for example, surgery versus palliative care. The surgeon writes the "best-case" and "worst-case" possible outcomes of each option on a linear continuum, as well as how likely these are for the particular patient before them. This includes explicit discussion of the quality of life that the patient can expect in each scenario. The patient can then visually determine how their personal preferences fit into the best or worst possible outcomes. While this approach takes additional time, the potential benefit of avoiding the post-operative conflicts discussed above is tremendous.

There is no question that a relationship with the patient improves both patient and surgeon satisfaction with difficult decisions, particularly surrounding complications and end of life care. Early engagement of the patient in conversations regarding advance care planning is critical [25]. By normalizing the language of these discussions during routine conversations with the patient, we will have an opportunity to learn the patient's preferences before often critical decisions must be made urgently. These conversations may also give the patient an opportunity to identify an appropriate surrogate decision-maker and to explicitly discuss their wishes with this person. A preoperative informed consent conversation is an optimal time to introduce this topic.

\section{Conclusion}

Today, patients increasingly prefer to participate in shared decision-making with their doctors. Under this pressure, the way in which we inform our patients and obtain consent for surgery must evolve. By taking time to include patient beliefs and values in this process, we may better align the expectations of our patients with our own expectations for their post-operative course. By doing this, we may prevent those painful situations when we are asked to withdraw 
treatment from a patient upon whom we have just operated. When the informed consent process is optimally undertaken, surgeons can be satisfied that we have done our very best for our patients.

\section{Compliance with ethical standards}

Conflicts of interest The authors declare no potential conflicts of interest.

\section{References}

1. Nabozny MJ, Kruser JM, Steffens NM et al (2016) Constructing high-stakes surgical decisions: it's better to die trying. Ann Surg 263:64-70

2. Sokol DK, Wilson J (2008) What is a surgical complication? World J Surg 32:942-944

3. Katz J (1998) Reflections on informed consent: 40 years after its birth. J Am Coll Surg 186:466-474

4. Etchells E, Ferrari M, Kiss A et al (2011) Informed decisionmaking in elective major vascular surgery: analysis of 145 surgeon-patient consultations. Can J Surg J Can Chir 54:173-178

5. Mulsow JJW, Feeley TM, Tierney S (2012) Beyond consentimproving understanding in surgical patients. Am J Surg 203: $112-120$

6. Deber RB, Kraetschmer N, Irvine J (1996) What role do patients wish to play in treatment decision making? Arch Intern Med 156:1414-1420

7. McKneally MF, Ignagni E, Martin DK, D'Cruz J (2004) The leap to trust: perspective of cholecystectomy patients on informed decision making and consent. J Am Coll Surg 199:51-57

8. Fried TR, Bradley EH, Towle VR, Allore H (2002) Understanding the treatment preferences of seriously ill patients. N Engl J Med 346:1061-1066

9. Steinhauser KE, Christakis NA, Clipp EC et al (2000) Factors considered important at the end of life by patients, family, physicians, and other care providers. JAMA 284:2476-2482

10. Cassell J, Buchman TG, Streat S et al (2003) Surgeons, intensivists, and the covenant of care: administrative models and values affecting care at the end of life. Crit Care Med $31: 1263-1270$
11. Nabozny MJ, Kruser JM, Steffens NM et al (2016) Patientreported limitations to surgical buy-in: a qualitative study of patients facing high-risk surgery. Ann Surg. doi:10.1097/SLA. 0000000000001645

12. Waisel DB (2000) The hazards of "hanging crepe" or stating overly pessimistic prognoses. J Clin Ethics 11:171-174

13. Siegler M (1975) Pascal's wager and the hanging of crepe. N Engl J Med 293:853-857

14. Bosk CL (2003) Forgive and remember: managing medical failure, 2nd edn. University of Chicago Press, Chicago

15. Wightman SC, Angelos P (2014) An organized approach to complex ethical cases on a surgical service. World J Surg 38:1664-1667

16. Sur MD, Angelos P (2015) Ethical issues in surgical critical care: the complexity of interpersonal relationships in the surgical intensive care unit. J Intensive Care Med 31:442-450

17. Schwarze ML, Bradley CT, Brasel KJ (2010) Surgical "buy-in": the contractual relationship between surgeons and patients that influences decisions regarding life-supporting therapy. Crit Care Med 38:843-848

18. Angelos P (2009) Complications, errors, and surgical ethics. World J Surg 33:609-611

19. Andrews LB, Stocking C, Krizek T et al (1997) An alternative strategy for studying adverse events in medical care. Lancet 349:309-313

20. Schwarze ML, Redmann AJ, Brasel KJ, Alexander GC (2012) The role of surgeon error in withdrawal of postoperative life support. Ann Surg 256:10-15

21. Casarett D, Ross LF (1997) Overriding a patient's refusal of treatment after an iatrogenic complication. $\mathrm{N}$ Engl $\mathrm{J}$ Med 336:1908-1910

22. Casarett DJ, Stocking CB, Siegler M (1999) Would physicians override a do-not-resuscitate order when a cardiac arrest is iatrogenic? J Gen Intern Med 14:35-38

23. Shanafelt TD, Balch CM, Bechamps G et al (2010) Burnout and medical errors among American surgeons. Ann Surg 251:995-1000

24. Kruser JM, Nabozny MJ, Steffens NM et al (2015) "Best Case/ Worst Case": qualitative evaluation of a novel communication tool for difficult in-the-moment surgical decisions. J Am Geriatr Soc 63:1805-1811

25. Sudore RL, Fried TR (2010) Redefining the "planning" in advance care planning: preparing for end-of-life decision making. Ann Intern Med 153:256-261 